\title{
Pedestrian Dead Reckoning with Wearable Sensors : a Systematic Review
}

\author{
Xinyu Hou and Jeroen Bergmann*
}

\begin{abstract}
Pedestrian Dead Reckoning (PDR) is the process of calculating one's current location by using the previously known position, and advancing that position over time using established or estimated speeds and trajectories (or alternatively stride lengths and directions). PDR plays an important role in modern life, including tracking locations of people and objects whenever GPS is not available. Self-contained PDR systems do not require an infrastructure, thus they can be used for rapid deployment in situations such as search and rescue, disaster relief or medical emergencies. Wearable sensors are often applied in self-contained PDR, but implementation varies in terms of the number, type and location of sensors used. Many algorithms are designed for PDR in order to reduce the error or drift of the final estimate, with various levels of success. There is a lack of comparison between these different methods and this systematic review of PDR for wearable devices provides a comprehensive overview that can inform further design optimizations. The aim of this paper is to assess the quality of all available PDR literature with a focus on wearable sensors. It provides an outline of the state-of-the-art in the field by comparing the accuracy of different sensor layouts and algorithms. Further directions of research are suggested based on these results. This study also highlights the need for more standardised and robust assessment protocols to capture real-world tracking performance of PDR methods.
\end{abstract}

Index Terms-Sensor networks, Navigation, Personal positioning, Local positioning systems, Pedestrian navigation

\section{INTRODUCTION}

$\mathbf{H}$ UMAN Position tracking technology, which provides information on the current position of an object or the location of an individual, has been rapidly developing over the last decennia. It has drastically changed modern life by offering information regarding the local position of things even during complex scenarios. Accurate tracking of human location is beneficial in everyday activities, such as providing navigation during the daily commute, but it can also be lifesaving in the case of disaster relief or during medical emergencies. This kind of tracking directly relates to the concept of Location Based Services (LBS). LBS includes e.g. navigation to a specific yet unfamiliar destination and it's used to help smart homes make better decisions. LBS even supports the management of personal health and emergency services, as well as contributes to the creation of systems for the next-generation of soldiers [1].

A wide range of sensors have been adopted for different pedestrian position tracking approaches. These approaches can be classified into two main categories: (i) dependent and (ii) self-contained methods. Dependent position tracking systems rely on external aiding signals, information or infrastructure, and include technologies such as global navigation satellite system (GNSS), received signal strength of wireless local area network (WLAN)/Bluetooth low energy (BLE) beacons/ultra wideband (UWB), radio frequency identification (RFID), magnetic maps, ambient measurements, floor plans or even just

This work was supported by EPSRC Impact Acceleration Account Award EP/R511742/1.

Xinyu Hou and Jeroen Bergmann are with the Department of Engineering Science, University of Oxford, Oxford, UK, OX1 3PJ (e-mail: jeroen.bergmann@eng.ox.ac.uk). generic maps. These dependent position tracking methods have been proven to be efficient and valid. They have been widely applied and have gained global traction. However, this method is not very applicable in scenarios where signals are severely affected by multipath propagation or when they are blocked by building materials. They also do not work when there is no specific infrastructure that can be used. It is relatively common that the limitations of these dependent methods surface during e.g. indoor, underground or wilderness tracking. The construction of infrastructure to facilitate dependent tracking also comes at a cost and demands continuous (electrical) energy to operate.

In contrast, self-contained position tracking does not rely on any infrastructure, which implies it could be used in any kind of environment. These infrastructure-independent methods for human localization are mainly based on Pedestrian Dead Reckoning (PDR), whilst utilizing on-body sensors. In navigation, PDR is the process of calculating one's current position by using a previously determined location, and advancing that location over time using known or estimated speeds [33]. Alternatively, course/stride length and direction can be used to determine the current position.

Most sensors utilised for PDR are small and wearable. They include Inertial Measurement Units (IMUs), accelerometers, gyroscopes, compasses, barometers, magnetometers, anemometers and on-body cameras to name some of the most popular types. These sensors are usually combined to create a multimodal system and are commonly mounted on the foot, wrist, waist, leg or the head. They are relatively low-cost and low energy, which makes them more attractive for use. PDR does not depend on any infrastructure, so it can be readily deployed in almost any situation. It is a particular 
useful application when conditions are rapidly changing. Thus, PDR is suitable during environmental emergencies, as the surroundings might be frequently changing. It also works well under smoky and dim-lit conditions, which makes it ideal for firefighting or rescue operations that could encounter similar environments.

Inevitably, measurement errors exist in sensor data and they could accumulated over time to reach a critical value. Highly accurate sensors are used in aviation and marine applications with the aim to keep the error at an acceptable level, whilst tracking positions over several hours. Yet, these sensor systems are too expensive and bulky for wearable use. The key user preferences for body-worn sensor systems is often the compact nature, ability to embed it in an everyday object and the simplicity to operate/maintain the system [?]. These specifications are captured perfectly in Micro ElectroMechanical Systems (MEMS). However, the open-loop integration of MEMS inertial sensor data is only suitable for a few minutes before drift dominates the signal. Thus, a lot of research is performed to develop effective algorithms that can reduce these errors. They range from Zero Velocity Updates (ZUPT) [8] to Extended Kalman Filter (EKF) [14] and Heuristic Drift Reduction (HDR) [50]. With the popularization of smartphones and the wide deployment of appropriate sensors in these smartphones, small low-cost and portable sensors have become more readily available for daily use. This systematic review specifically explores the obtained accuracy of PDR when wearable sensors are utilised.

Several review studies have already been published regarding PDR methods, but all of them have been limited in scope. For example, [1], [2] are surveys of the research status of PDR and only include inertial sensors; [3] only focused on step length estimation methods; [4] explored PDR just using inertial and magnetic sensors and [5] reviewed PDR specifically for mass market applications. Despite these numerous reviews in PDR, none of them performed a comprehensive analysis on PDR across a range of wearable sensors. More importantly, none of them conducted a systematic search or included a quality assessment of the research. In this paper we systematically reviewed all studies related to PDR with wearable sensors. This study provides an overview of the used sensor placement, applied algorithms and adopted evaluations.

\section{Methods}

\section{A. Search Strategy}

The Preferred Reporting Items for Systematic Reviews and Meta-Analyses (PRISMA) [6] was used to structure the content provided below. The literature search was conducted using these three databases: (i) PubMed, (ii) Web of Science and (iii) IEEEXplore. A total of 18 keywords were selected and combined with Boolean operators to obtain all papers related to PDR and wearable sensors. All papers published before February 2020 were included in this systematic search.

The search strategy applied consisted of the following keyword combination: ("dead reckoning" OR "position tracking" OR localization OR "position monitoring") AND ("physical activity" OR sports OR walking OR running OR pedestrian)
AND (accelerometer OR "Inertial Measurement Unit" OR Gyroscope OR Magnetometer OR "Body Sensor Network" OR "wearable sensor" OR "wearable sensors") NOT GPS NOT wifi

\section{B. Study Selection}

A variety of sensors could be used for PDR, but only studies using wearable sensors were included. Wearable sensors consisted of sensors, such as IMUs, gyroscopes, magnetometers, compasses, Electromyography(EMG) sensors, sonar, on-body cameras, barometers and anemometers. Studies that used sensors that were not attached to the body or that relied on other infrastructures (for example GPS, Wi-Fi, RFID, UWB, Bluetooth beacon, radio, etc.) were excluded. Studies amending the trajectories with previously known information or data were also excluded. Therefore, research that relied on information taken from an earlier established map, floor plan, magnetic fingerprints, light fingerprints or similar constructs were not included.

The inclusion criteria for articles were:

1. It must be written in the English language.

2. It must be peer reviewed.

3. It must contain an outcome measure - methodology alone does not suffice.

4. It must explore a PDR method using wearable sensors.

5. It must report a quantitative tracking error.

The characteristics and data extracted from each study consisted of (i) year of publication, (ii) algorithm used, (iii) type, amount and position of sensors, (iv) error and total travel distance.

All eligible studies that were not accessible through library services were attempted to be obtained by contacting the corresponding author.

\section{Quality Assessment}

The quality assessment of each paper followed the questions of the adapted Specialist Unit for Review Evidence (SURE) to assist with the critical appraisal of the studies [7]. The checklist was modified to better fit the field of this systematic review. The modified checklist included 8 questions, which can be found in Table I.

\section{Results}

A total of 877 records were identified by searching the aforementioned databases. A total of 265 duplicates were removed from the list. The titles and abstracts of 612 remaining papers were subsequently screened by both authors. The full manuscript was assessed for the 307 cases that remained after review of the title and abstract. The 307 articles were obtained using the University library system, online resources and by contacting corresponding authors. This led to the full review of 301 papers, as not all articles were obtainable. Finally, 145 studies met the inclusion criteria and were further appraised (see Fig. 1). A sample summary of the study characteristic is presented in Table II. The complete table for characteristic summary and quality assessment can be found in the supplementary materials. 
TABLE I

MODIFIED SURE CRITICAL APPRAISAL CHECKLIST FOR SYSTEMATIC REVIEW AND 9 SAMPLES OF QUALITY SCORES

\begin{tabular}{|c|c|c|c|c|c|c|c|c|c|c|}
\hline Item & Checklist & $\begin{array}{l}\text { Wang } \\
{[13]}\end{array}$ & $\begin{array}{l}\text { Shin } \\
{[17]} \\
\end{array}$ & $\begin{array}{l}\text { Shi } \\
{[18]} \\
\end{array}$ & $\begin{array}{ll}\mathrm{Li} \\
{[19]} \\
\end{array}$ & $\begin{array}{l}\text { Guo } \\
{[20]} \\
\end{array}$ & $\begin{array}{l}\text { Park } \\
{[21]} \\
\end{array}$ & $\begin{array}{l}\text { Lee } \\
{[22]} \\
\end{array}$ & $\begin{array}{l}\text { Yang } \\
{[25]}\end{array}$ & $\begin{array}{l}\text { Cardarelli } \\
{[70]} \\
\end{array}$ \\
\hline 1 & $\begin{array}{l}\text { Does the study address a } \\
\text { clearly focused question? }\end{array}$ & 1 & 1 & 1 & 1 & 1 & 1 & 1 & 1 & 1 \\
\hline 2 & $\begin{array}{l}\text { Do the authors discuss how } \\
\text { they decided which method to } \\
\text { use? }\end{array}$ & 1 & 1 & 1 & 1 & 1 & 1 & 0 & 1 & 0 \\
\hline 3 & $\begin{array}{l}\text { Is there sufficient detail regard- } \\
\text { ing the methods used? }\end{array}$ & 0 & 0 & 1 & 1 & 1 & 0 & 1 & 0 & 1 \\
\hline 4 & $\begin{array}{l}\text { Is the data collection method } \\
\text { well described? }\end{array}$ & 0 & 1 & 1 & 0 & 1 & 0 & 0 & 1 & 1 \\
\hline 5 & $\begin{array}{l}\text { Are the explanations for the } \\
\text { results plausible and coherent? }\end{array}$ & 0 & 1 & 1 & 0 & 1 & 0 & 0 & 1 & 1 \\
\hline 6 & $\begin{array}{l}\text { Are the results of the study } \\
\text { compared with those from } \\
\text { other studies? }\end{array}$ & 1 & 0 & 1 & 0 & 1 & 0 & 0 & 1 & 0 \\
\hline 7 & $\begin{array}{l}\text { Did the authors identify any } \\
\text { limitations? }\end{array}$ & 0 & 0 & 1 & 0 & 1 & 0 & 1 & 0 & 0 \\
\hline 8 & Was ethical approval sought? & 0 & 0 & 0 & 0 & 0 & 0 & 0 & 0 & 1 \\
\hline
\end{tabular}

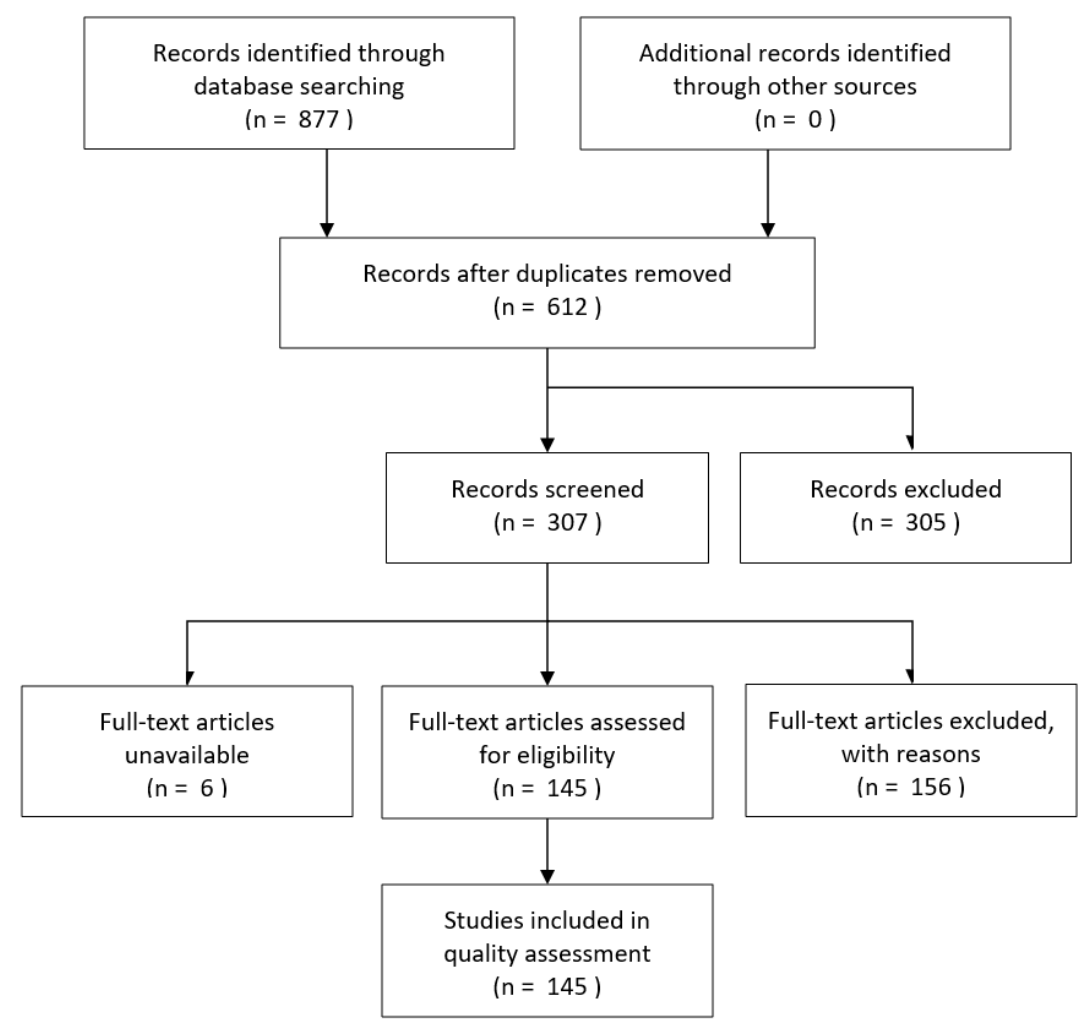

Fig. 1. PRISMA flowchart of systematic review. The process consisted of identification, duplicate removing, screening and inclusion of relevant papers.

\section{DESCRIPTIVE SUMMARY OF RESULTS}

\section{A. Sensor types and layouts}

A variety of different sensors were applied in the studies that were identified. The difference in the type, the amount and the placement on the body of the sensors also led to contrasting algorithm designs.

1) Types: The IMU was the most common used sensor $(n=81)$ across all the studies. It consisted of a triaxial accelerometer, a triaxial gyroscope, and sometimes a triaxial magnetometer. It was reported that the data from the magnetometer was not always a reliable source for heading estimation, due to the existence of soft and hard magnetic disturbance in the environment. This was especially true for indoor environments. Only a few studies $(n=32)$ adopted magnetometers for azimuth calculation.

Some studies also used other wearable sensors to increase the tracking accuracy, beyond the application of IMUs. Cameras were utilized to extract features of the environment [25], whilst others used pressure sensors under the shoe to detect heel-strike and toe-off points that could aid in the step 
TABLE II

SAMPLE OF STUDY CHARACTERISTICS SUMMARY

\begin{tabular}{|c|c|c|c|c|c|c|c|c|}
\hline $\begin{array}{l}\text { First author, } \\
\text { year of pub- } \\
\text { lication }\end{array}$ & Sensor layouts & Algorithms & $\begin{array}{l}\text { No. of } \\
\text { subjects }\end{array}$ & $\begin{array}{l}\text { Mean error } \\
(\mathrm{m})\end{array}$ & $\begin{array}{l}\text { Total travel dis- } \\
\text { tance }(\mathrm{m})\end{array}$ & Accuracy & Environm & Dimensio \\
\hline $\begin{array}{l}\text { Wang, } 2016 \\
{[13]}\end{array}$ & $\begin{array}{l}\text { A smartphone, a foot- } \\
\text { mounted IMU }\end{array}$ & $\begin{array}{l}\text { zero velocity update, } \\
\text { Indoor landmark detec- } \\
\text { tion, online magnetic } \\
\text { trajectory matching }\end{array}$ & 1 & 2.55 & - & $\begin{array}{l}80 \text { percentile } \\
\text { localization } \\
\text { accuracy of } \\
1.4 \mathrm{~m}\end{array}$ & Indoor & $2 \mathrm{D}$ \\
\hline $\begin{array}{l}\text { Shin, } 2014 \\
{[17]}\end{array}$ & A smartphone & $\begin{array}{l}\text { motion recognition, } \\
\text { ZUPT, KF, SVM }\end{array}$ & 3 & 2.25 & 133.2 & $5.00 \%$ & Indoor & $3 \mathrm{D}$ \\
\hline $\begin{array}{ll}\text { Shi, } & 2019 \\
{[18]} & \end{array}$ & $\begin{array}{l}\text { An IMU mounted on } \\
\text { foot }\end{array}$ & $\begin{array}{l}\text { zero velocity update } \\
(\mathrm{ZVU}) \text {, Gait Phase De- } \\
\text { tection Algorithm, KF }\end{array}$ & 1 & $\begin{array}{l}0.74 \text { indoor, } \\
2.33 \text { outdoor }\end{array}$ & $\begin{array}{l}\text { 103.86indoor, } \\
\text { 402.7outdoor }\end{array}$ & $\begin{array}{l}0.73 \% \text { indoor, } \\
0.58 \% \text { outdoor }\end{array}$ & $\begin{array}{l}\text { Indoor + } \\
\text { Outdoor }\end{array}$ & $2 \mathrm{D}$ \\
\hline $\begin{array}{l}\mathrm{Li}, \\
{[19]}\end{array}$ & $\begin{array}{l}\text { A foot-mounted IMU, } \\
\text { magnetometer }\end{array}$ & $\mathrm{KF}$, peak detection & 1 & 4.27 & 400 & $1.00 \%$ & Outdoor & $2 \mathrm{D}$ \\
\hline $\begin{array}{l}\text { Guo, } 2015 \\
{[20]}\end{array}$ & A Foot-Mounted IMU & ZARU, ZUPT & 1 & 0.88 & 90.48 & $1.00 \%$ & Indoor & $2 \mathrm{D}$ \\
\hline $\begin{array}{ll}\text { Park, } & 2012 \\
{[21]} & \end{array}$ & A waist-worn IMU & HDR & 1 & - & 64.48 & $3.00 \%$ & Indoor & $2 \mathrm{D}$ \\
\hline $\begin{array}{l}\text { Lee, } 2002 \\
{[22]}\end{array}$ & $\begin{array}{l}\text { A biaxial accelerome- } \\
\text { ter and the gyroscope } \\
\text { located in one trouser } \\
\text { pocket; a digital com- } \\
\text { pass and a microcon- } \\
\text { troller attached to the } \\
\text { middle of the user's } \\
\text { waist. }\end{array}$ & fuzzy logic algorithm & 8 & - & 90 & $8.20 \%$ & Indoor & $3 \mathrm{D}$ \\
\hline $\begin{array}{l}\text { Yang, } 2017 \\
{[25]}\end{array}$ & $\begin{array}{l}\text { A smartphone (cam- } \\
\text { era) }\end{array}$ & $\begin{array}{l}\text { Hybrid Orientation Fil- } \\
\text { ter }\end{array}$ & 5 & 0.83 & 207 & $0.41 \%$ & Indoor & $2 \mathrm{D}$ \\
\hline $\begin{array}{l}\text { Cardarelli, } \\
2019 \text { [70] }\end{array}$ & A foot mounted IMU & KF, ZUPT & 1 & 0.96 & 43.16 & $2.22 \%$ & Indoor & $2 \mathrm{D}$ \\
\hline
\end{tabular}

detection [26]. Electromyography (EMG) sensors were also applied to get EMG signals of the Gastrocnemius for both step detection and step length estimation [27]. Other methods that were reported leveraged a compass to get geomagnetic information [28], used a barometer to estimate the vertical moving distance [29], used an anemometer to estimate walking speed [30], used an ultrasonic sensor to estimate step length [31] or use of a laser scanner to calculate relative distances [32]. A large portion of the studies that were included $(n=49)$ applied smartphones as their data collection and measuring device.

2) Layouts: The sensors were mounted on a variety of locations on the body. Studies with a single IMU, most frequently mounted the sensor on one of the shoes [33]. Another commonly selected placement was the waist [34]. Studies with multiple IMUs preferred to mount them on the lower limb and trunk [35]. This included placement on the pelvis, thighs, shanks and feet. There were studies that had an even larger network of IMUs available and they also placed sensors on the upper limb in addition to the aforementioned locations [36]. For the upper limb, sensor placement consisted of the shoulder, upper and lower arms. Studies relying on a smartphone usually resorted to having the sensor near the hand or in the pocket at the front/back of the trousers, as well as the pocket located on the shirt/jacket [37]. Holding the smartphone in the hand allowed for a further breakdown based on the specific task that was performed on the phone. Separation of phone tasks was used to help with the data analysis and activities consisted of texting, swinging and calling [38]. The study with the most diverse type of sensors, placed a camera on the chest, one IMU on the head, one on the back and one on the shoe, whilst also relying on sonar with the user carrying a computer on the back [39].

\section{B. Algorithms}

To robustly estimate the trajectory of pedestrians, the designed algorithms should be fit to operate correctly under a range of different conditions. Ideally, they should also be able to work for a range of sensors, placements, motions, people and environments. Algorithms were subdivided to better cluster the outcomes from the studies. The PDR systems are divided into categories that are described below [1]:

INSs (Inertial Navigation Systems): An INS is a system that tracks position by estimating the full 3D trajectory of the sensor at any given moment. INSs are based on the theory that the first integration of the acceleration signal measured by the accelerometer produces the real-time velocity and the successive integration of velocity then gives the real-time displacement. The real-time attitude is produced by integration of the angular velocity, which is measured by the gyroscope. The accumulation of displacement and attitude is subsequently used. However, the integration error due to noise, bias and other disturbances in this measurement is not negligible and can increase quickly. There are various algorithms presented in the papers to correct or compensate for these errors.

SHSs (Step-and-Heading Systems): An SHS is specific to pedestrians, estimating position by accruing distance, heading vectors representing either steps or strides. In certain cases a 3D trajectory is not required for a specific tracking scenario and the 2D navigation in the horizontal plane using step 
vectors, rather than complete limb trajectories, is sufficient. SHSs output a series of step vectors by detecting each step of the pedestrian, estimating the length and direction of it, and finally integrating every step to obtain a complete trajectory.

There are also other techniques available for data analysis and these will now be discussed.

1) Zero velocity update (ZUPT) and zero-angular rate update (ZARU): This approach relies on the idea that the amount of drift on the signal could be reduced if the integration loop can be closed using external constrains. The Zero Velocity Update (ZUPT) and Zero Angular Rate Update(ZARU) both make use of PDR constrains [14], [33], [35]. ZUPT was first used by Foxlin et al in the NavShoe project and good results were reported for gait [8]. Human walking consist of two distinct phases (stance and swing phase). The foot is in contact with the ground during the stance phase, which provides a useful reference point as the angular and linear velocity of the foot is close to zero during large portions of the stance phase. This information can be detected using the aforementioned algorithms and allows for separation of individual steps. The ability to label each step provides a method to reduce longterm accumulation of errors. However, ZUPT and ZARU are only suitable for tracking systems that consist of IMUs mounted on the foot.

2) Magnetic information: Geomagnetic fields cover the earth's surface and this information can sensed using magnetometers. Magnetometers measure the direction of the local magnetic field and can help derive the absolute heading direction. This information can provide a useful correction for the inevitable heading drift, which arises from the integration of gyroscopic output. However, the geomagnetic field is not always uniform and can be easily disturbed by hard or soft magnetic interferences. This means that the heading information produced from magnetometers does not always provide a stable directionality. Only a few studies apply magnetometers and they have specially designed algorithms to deal with these magnetic disturbances. These algorithms aim to calibrate, compensate or rely on the disturbances in order to create a more accurate position estimate. The magnetic measurements can also benefit from the application of a Kalman filter. As an example, Ashkar [14] estimated the magnetic field offset, which is then treated similar to the gyroscope and accelerometer biases within the measurement model. Another common correction algorithm depends on fusing gyroscope output with the compass information. This works well because of their complementary error characteristics. The gyroscope has a accumulation of errors in the long-term orientation, while magnetometers are subjected to short-term orientation issues [15]. Finally, a Quasi-Static magnetic Field (QSF) can also be used to detect particular QSF periods during movement. The changes in magnetic field components during these periods can be compared with the angular rates of the overall sensor unit. Thus, providing further information that can help compensate for any arising errors [16].

Some researches exploit the fact that the magnetic field is not uniform. This provides the opportunity to use the sensor output for "fingerprinting". They collect magnetic field maps before tracking and then apply a matching algorithm to estimate position during real-time data collection based on the previously obtained map [10]-[12]. The concept of matching to an established map does yield good results, but articles that employ this technique are excluded from this review since they require predetermined information. Interestingly enough there is a study that does take advantage of the magnetic anomalies, but does not rely on collecting a historic map before data collection. This study employs a process that consists of collecting magnetic anomalies in real-time to calibrate the PDR location without the need of deploying any additional infrastructure or performing offline site surveying [13]. Shin et al. use a support vector machine (SVM) for the heading estimation by recognising the consistent patterns in magnetic fields when orientated in a certain direction [17].

3) Gait cycle detection: Gait cycle detection was indirectly discussed in the ZUPT and ZARU section. However, step detection is more broadly used and it can serve different purposes, including step counting. Wu et al. proposed that gait division strategies could fall into two categories: (i) the abbreviated and (ii) the detailed strategy [2]. The abbreviated strategy consist of simply dividing the gait cycle into only one or two events. For example, dividing it into a stance and swing phase [18]. The detailed division strategy refers to separating the gait cycle into three or more phases. One possible option is the division of gait into four main stages such as a contact phase, mid-stance phase, propulsive phase and swing phase [19]. Other breakdowns of the gait cycle in the temporal domain are possible.

Detection of these phases can be facilitated by thresholdbased algorithms, which are typically used for stance detection. Corresponding gait phases can also be determined by measuring if a specific set of features are part of the threshold set. Guo et al. set three thresholds to split-up the gait cycle. They used the magnitude and variance of the acceleration, as well as the magnitude of the angular rate to estimate the stance phase [20].

Another way of doing it is relying on a peak detection method to recognise gait and steps by detecting the maximum peak of the acceleration output signal [19]. Other popular methods in the time domain include a zero crossing detection method [21], the application of fuzzy logic [22] or applying autocorrelations [23]. These methods are usually based on accelerometer signals and the data is often pre-processed by using a low-pass filter to reduce noise. Approaches in the frequency domain have also been explored and include a ShortTime Fourier Transform (STFT) [24] or a continuous/discrete wavelet transforms (CWT/DWT) [41].

Feature clustering approaches that employ machine learning algorithms have started to become more popular. Hidden Markov Models (HMMs) can be trained by two state HMM with Gaussian emissions in an unsupervised fashion using the Viterbi algorithm and K-means clustering to classify activities based on features extracted from inertial measurements in both the time and frequency domains. Zhang et al. used a HMM as a gait detector during dynamic and fast gait speeds in [28]. Edel and Koppe used Bidirectional Long Short-Term Memory Recurrent Neural Networks (BLSTM-RNNs) to accomplish a 3D step recognition approach [40]. 
Finally, step detection can also be accomplished using sensors that do not contain inertial sensing capabilities. Chen et al. used pressure sensors embedded in the sole of the shoe [26], whilst Zizzo et al. used two position-sensitive detectors (PSDs) mounted on the toe and heel of the shoe [31] for phase detection. Chen et al. researched the ability of two EMG sensors that were attached to the calves [27].

4) Step length estimation: One of the simplest approaches for step length estimation is to set the step length as a constant value. An average value can be set when people walk at their own "natural" pace. The detection of the step frequency can then be combined with this pre-set step length value. Issues arise when subjects are starting to deviate from this value. This can occure when they change their activity to running, turning or even when they start to walk in groups. A dynamic step length estimation method is more appropriate in these situations. One such model is the Weinberg model [51], which assumes that the vertical bounce is proportional to the step length. The Kim model [52] aims to associate step length and the average acceleration that occurs during a step. Scarlet's approach [53] focuses on the spring variation in the steps of different people, or in the steps of one person walking at different velocities. Methods have also been introduced that build a model using linear methods [54] or SC-based methods [55].

Direct measurement of step length can also be accomplished with non-inertial sensors. Zizzo used five ultrasound receivers on the left foot and two ultrasound transmitters on the right foot to measure the step displacement [31].

5) Heading estimation: Heading is usually estimated by gyroscopes, which output a relevant angular velocity. The integration of gyroscope signals provide the heading change and as previously mentioned the heading drifts can then be limited by e.g. ZUPT or ZARU. Yet, ZUPT and ZARU are not very suitable when the sensors are not placed on the foot. Thus, sometimes headings need to be corrected using other processes. For instance, Heuristic Heading Reduction (HDR) can limit the constant drift based on the assumption that when roads are straight, the pedestrian heading should remain unchanged [50]. The suitability of this method is decreased when reality is starting to deviate from this assumption.

6) Data fusion: Filtering algorithms that integrate multiple observations are important in PDR systems with multiple sensors. The most commonly used algorithm among the presented studies is the Kalman Filter (KF) [41]. This is a recursive Bayesian filter, known to be an optimal filter for Gaussian linear systems. KF uses a series of measurements that are observed over time (containing statistical noise and other inaccuracies) to produce estimates of unknown variables. These estimates tend to be more accurate than those based on a single measurement alone. An output from the filter is obtained by estimating the joint probability distribution over the variables for each given time frame. Many studies did not use the original KF, but applied an extensions or generalizations of it. The following filters were reported; the Unscented Kalman Filter (UKF) [42], Cascaded Kalman Filter (CKF) [43], Extended Kalman Filter (EKF) [14], quaternion-based extended Kalman Filter [44], Adaptive Kalman Filter (AKF)
[45] or Robust Adaptive Kalman Filter (RAKF) [46]. These were some of the more common adaptations, but other filter methods were also found. A Complementary Filter (CF) is such a filter. It is applied to make accelerometer and gyroscope data complement each other in order to get the initial attitude [47]. The Particle Filter (PF) is another one. This filter is a numerical approximation to a Bayesian filter [48] and is often used in walking length and direction estimation. PF consists of many 'particles' that represent possible positions and headings with a weighted value indicating the probabilities of each. Adjusting these values is an iterative process that consist of three steps (update, correct and resample) [49].

$\mathrm{Wu}$ et al. divided the filtering methods into direct and indirect estimations. The direct estimation is a method where the filter directly outputs the final tracking data, whilst the indirect estimation refers to the fact that the filter estimates the errors of the tracking data before compensating for errors in the integration results.

7) Mode Classification: It is essential to keep in mind that pedestrians do not always walk with a regular pace. Gait itself can be affected by environmental or behavioral situations. Inderst et al. classified patterns as walking, standing, ascending and descending stairs before estimating any positions [34]. Hussain also classified the recorded signals into different motion states that included walking, running and stopping [57].

The classification of modes can go beyond mobility classification, whenever a smartphone is used as the primary data collection system. Different classes can be defined in terms of how the phone is carried or held. Errors can occur if this is not taken into account, since there are various "holding" locations and carrying modes for smartphones [38]. The phone might be placed in the hand, located in the front trousers pocket, front shirt/jacket pocket or back trousers pocket [37]. The ability to know where the phone is located creates an additional relevant layer in the process of estimating the position of the user.

Adopting these diverse tracking strategies for different modes aims to increase the overall tracking accuracy. Appropriate models are needed to correctly classify these modes and most papers make use of machine learning algorithms to do so. The classification models encountered in this review are Long Short-Term Memory (LSTM) [57], Support Vector Machine (SVM) [58], Muti-layer perceptrons (MLP) [59], Probabilistic neural network classifiers (PNN) [60] and Random Forest models [61].

8) Simultaneous Localisation and Mapping (SLAM): The Simultaneous Localisation and Mapping (SLAM) proposition combines the localisation problem with a map estimation. It iteratively builds a map of the environment and localizes the user within this map. Kaiser and Diaz investigated PocketSLAM by combining PocketNav with FootSLAM [63]. Hardegger et al. proposed ActionSLAM, which used locationrelated actions as landmarks [64].

\section{Evaluation}

The results shows that there was no standard procedure implemented across the papers to evaluate PDR accuracy. The 
process by which the algorithm was evaluated differed greatly between the studies. Moreover, the underlying ground truth regarding the positions that an individual passed over time also varied between the experiments. This lack of consistency makes it harder to compare the performance of each algorithm. Ideally, the exact same procedure and setup is used to create a condition in which only the selected algorithms differ between measurements.

The testing environments of the studies were heterogeneous and thus a one-to-one mapping of accuracies between techniques was not possible. One area of divergence in terms of environment related to the experiments taking place either in- or outdoor. A total of 20 studies tested their systems in an outdoor environment, 108 papers stayed indoors and 17 studies tested both in- and outdoor. For indoor evaluations, the ground truths of tracking trajectories often came from optical motion capture systems [31], multiple cameras [64], labels along a certain route with a downwards-facing handheld camera [40], center of the route on a floor plan [65], laser distance meters [66], commercial robot localization system including an infrared camera and a set of passive landmarks placed on the ceiling [67] or a general motion tracking system (MTS) [68]. All these data collection systems come with their own levels of accuracy. They also limit the kind of activities that could be performed, as well as the distances and patterns that can be verified with these devices. For the outdoor evaluations, the ground truths mainly came from a GPS receiver [69] or simply by referencing a pre-determined trajectory [18]. The same considerations need to be taken into account for the gold reference systems that are used in an outdoor environment. Determining if a certain accuracy of a PDR technique is generalizable to other scenarios is limited by the initial experimental setup. Creating results in terms of accuracy that translate easier to real-world deployments involves the inclusion of more realistic testing conditions. It is suggested that more work is done to reflect specific scenarios of interest during the testing of PDR approaches.

Tracking was performed in $2 \mathrm{D}$ or $3 \mathrm{D}$. The $2 \mathrm{D}$ PDR estimated the trajectory of a person in the horizontal plane, while 3D PDR provided an extra vertical dimension for calculating the current position. Most studies $(n=105)$ tracked people in 2D. However, the 3D methodology was still explored in 40 studies. The dimensionality wasn't the only thing that differed in terms of the paths that were set in each study. There was also a big dissimilarity between the total travel distances in the experimental setups. The longest walking distance for one particular test was $4,029 \mathrm{~m}$ [73], whilst the shortest was found to be $6 \mathrm{~m}$ [74].

Some studies were conducted to evaluate the robustness of the system, for example by testing trajectories with different shapes [71]or testing with smartphones of different brands [72]. This is a suitable notion to investigate, as it connects well to the need of more real-world evidence for PDR. Including variability into the scientific research allows for a broader interpretation. The same benefit can be claimed for using multiple volunteers in order to assess the PDR performance. People do produce changed gaits under different conditions and capturing a more representative groups of users will contribute to a potential greater uptake of this technology. Currently, the total number of test subjects varied considerable between studies. The majority of studies had only one participant ( $\mathrm{n}=96)$, but 15 studies had more than 10 subjects and the maximum number of subjects recruited for a single paper was 30.

\section{D. errors}

No standard method for computing the error was adopted across all studies, which echoes the issue that was highlighted for the experimental setup. Researchers showed their tracking accuracy through a range of error representations. They consisted of calculating the end-to-end error, determining a position/distance error, defining the position error rate, giving a return position error, as well as reporting deviations on three axis and calculating the root mean square error or standard deviation. A percentage error was calculated in this review to provide a better comparison between the research studies. The smallest error found was $0.07 \%$, while the largest error is $10.07 \%$. The median of all the errors reported for PDR was around $1 \%$.

\section{DISCUSSION}

This paper performed a systematic review and quality assessment of studies that discussed PDR with wearable sensors. The selected papers were analysed across several factors to provide an easy comparison for those interested in this topic. The sensor placement, applied algorithm and evaluation methods were reported across all included studies. It is difficult to objectively compare the different PDR systems, as the experimental conditions varied along with the error metric that was used to quantify the performance of the technique. The field could benefit from adopting a testing method that can be applied more widely whenever systems and techniques need to be compared. The testing setup should aim to represent realworld scenarios in order to be able to generalise the results to potential functional applications. Fig 2 presents an overview of the sensor placements and the average error for each position. It shows that attaching sensors on the foot may gain a better result, which is likely to be mainly caused due to the adoption of ZUPT or ZARU. These are two often used algorithms and can only be used in PDR with foot-mounted sensors. Filters like KF are also essential to PDR because of the commonly applied multi-sensor methods. As for the type of sensors, almost all the studies were using IMUs, which is recognised as the dominant type of sensors for detecting movements. It is expected that IMUS will stay one of the core technologies for PDR in the future.

As previously stated, many studies relied on IMUs to perform PDR. In [75], Zhao, et al. designed an approach to reduce the heading drift for foot-mounted sensors by implementing dual-gait analysis and multi-sensor fusion. The setup consisted of a dual-sensor configuration with one IMU on each foot. This study yielded a relative small position error of $0.76 \%$. It introduced a novel implementation of dual-gait analysis for multi-sensor fusion, relying on a Kalman-type filter. The advantage is that it does not require a personalised 
parametric adjustment or calibration for each individual user. It also primarily depends on the gyroscope signal, which has a lower signal-to-noise ratio (SNR) than an accelerometer, due to the specificity of the relevant foot movement. However, a foot-mounted system might not always be functional for prospective users. Another study [76] designed an indoor position estimation algorithm using an IMU located in a smartphone. They created a pitch-based step detection method that combined the accelerometer and gyroscope signals, with an additional sensor fusion heading estimation based on the gyroscope and magnetometer. A displacement error of $0.61 \%$ was obtained in the study, showing that it could outperform a conventional method in terms of error by factor of 2 . This described method is easy to implement and could be used in real- time navigation. However, the results might not generalise well as it was only tested once on a single subject. The described experiment was performed indoor under ideal conditions requesting the subject to walk on an even floor. How these results translate to surroundings that are less ideal remains unclear. One study that did perform tests under more diverse scenarios showed some promising results. In [77], Qiu, et al. proposed a multi-sensor fusion PDR method using EKF, zero velocity updates (ZVU) and a clustering algorithm for stance phases distinction. This study completed the most diverse set of experiments out of all papers that were included in this review. It reached rather competitive errors of less than $1 \%$, despite the method being tested under more challenging conditions than most other studies. Indoor and outdoor scenarios were tested and they included walking on level ground, as well as climbing stairs. This outcome provided some insights into the robustness of this method.

Interestingly enough, among all 145 papers, only one of them had ethical approval in place. This poses a serious question in how some of this data was collected and stored. It is strongly recommended that reviewers and editors of journals only accept human-based studies that have obtained the relevant ethical approval.

The summarised results shows that across all papers a relatively high level of accuracy was reached (1\%). However, it is hard to say if this result had any external validity. Realworld scenarios are very different from most of the testing conditions reported in the articles and it is very probable that the error significantly increases when these systems move outside the lab. People may run, jump, turn, slide or stumble in daily activities, and the ground we walk on could be uneven, rugged or inclined. Position tracking in these environments is far more complex than walking on a smooth surface with a constant pace. Pushing system testing to include more challenging conditions will help create the next generation of PDR systems.

\section{ACKNOWLEDGEMENT}

This paper was financially supported by the EPSRC Impact Acceleration Account Award EP/R511742/1.

\section{REFERENCES}

[1] R. Harle, "A Survey of Indoor Inertial Positioning Systems for Pedestrians," in IEEE Communications Surveys \& Tutorials, vol. 15, no. 3, pp. 1281-1293, Third Quarter 2013. doi: 10.1109/SURV.2012.121912.00075

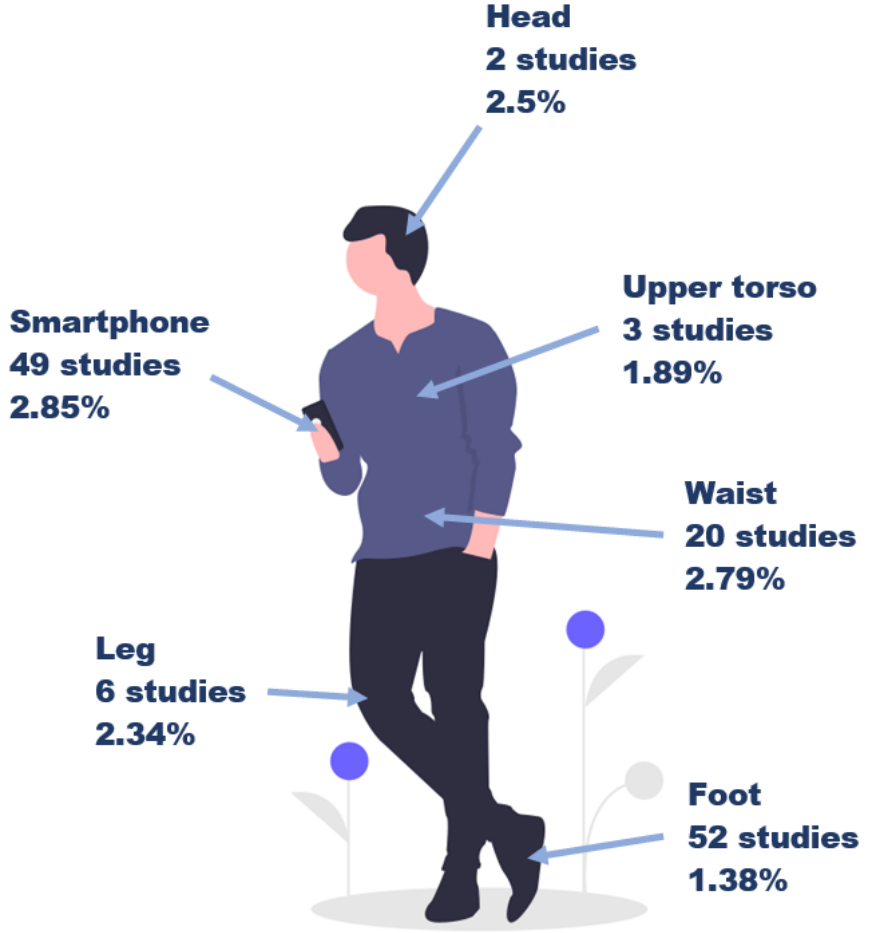

Fig. 2. Average error of different sensor mounted positions.

[2] Y. Wu, H.-B. Zhu, Q.-X. Du, and S.-M. Tang, "A survey of the researchstatus of pedestrian dead reckoning systems based on inertial sensors,'Int. J. Autom. Comput., vol. 16, no. 1, pp. 65-83, 2019.

[3] L. E. Díez, A. Bahillo, J. Otegui and T. Otim, "Step Length Estimation Methods Based on Inertial Sensors: A Review," in IEEE Sensors Journal, vol. 18, no. 17, pp. 6908-6926, 1 Sept.1, 2018.

[4] F. W. Gobana, "Survey of Inertial/magnetic Sensors Based pedestrian dead reckoning by multi-sensor fusion method," 2018 International Conference on Information and Communication Technology Convergence (ICTC), Jeju, 2018, pp. 1327-1334.

[5] A. Correa, M. Barcelo, A. Morelland, and J. Vicario, "A review of pedestrian indoor positioning systems for mass market applications," Sensors, vol. 17, no. 8, p. 1927, 2017.

[6] D. Moher, A. Liberati, J. Tetzlaff and D. G. Altman, "Preferred Reporting Items for Systematic Reviews and Meta-Analyses: The PRISMA Statement," International Journal of Surgery, vol. 8, no. 5, pp. 336-341, 2010.

[7] Specialist Unit for Review Evidence (SURE) 2015. Questions to assist with the critical appraisal of qualitative studies available at: http://www.cardiff.ac.uk/specialist-unit-for-reviewevidence/resources/critical-appraisalchecklists

[8] E. Foxlin, "Pedestrian Tracking with Shoe-Mounted Inertial Sensors," IEEE Computer Graphics and Applications, vol. 25, no. 6, pp. 38-46, Nov. 2005.

[9] S. Rajagopal, "Personal dead reckoning system with shoe mounted inertial sensors," In Master of Science Thesis, Stockholm, Sweeden, pp. $1-45,2008$

[10] H. J. Jang, J. M. Shin and L. Choi, "Geomagnetic Field Based Indoor Localization Using Recurrent Neural Networks," GLOBECOM 2017 2017 IEEE Global Communications Conference, Singapore, 2017, pp. 1-6.

[11] C. Huang, S. He, Z. Jiang, C. Li, Y. Wang and X. Wang, "Indoor positioning system based on improved PDR and magnetic calibration using smartphone," 2014 IEEE 25th Annual International Symposium on Personal, Indoor, and Mobile Radio Communication (PIMRC), Washington, DC, 2014, pp. 2099-2103.

[12] G. Wang, X. Wang, J. Nie and L. Lin, "Magnetic-Based Indoor Localization Using Smartphone via a Fusion Algorithm," in IEEE Sensors Journal, vol. 19, no. 15, pp. 6477-6485, 1 Aug.1, 2019.

[13] Q. Wang, H. Luo, F. Zhao and W. Shao, "An indoor self-localization algorithm using the calibration of the online magnetic fingerprints and 
indoor landmarks," 2016 International Conference on Indoor Positioning and Indoor Navigation (IPIN), Alcala de Henares, 2016, pp. 1-8.

[14] R. Ashkar, M. Romanovas, V. Goridko, M. Schwaab, M. Traechtler and Y. Manoli, "A low-cost shoe-mounted Inertial Navigation System with magnetic disturbance compensation," International Conference on Indoor Positioning and Indoor Navigation, Montbeliard-Belfort, 2013, pp. 1-10.

[15] Ladetto, Q.; Merminod, B. An Alternative Approach to Vision Techniques: Pedestrian NavigationSystem Based on Digital Magnetic Compass and Gyroscope Integration. InProceedings of the6th World MultiConference on Systemics, Cybernetics and Informatics, Orlando, FL, USA, 14-18July 2002; pp. 145-150

[16] M. H. Afzal, V. Renaudin, and G. Lachapelle, "Use of Earth's magneticfield for mitigating gyroscope errors regardless of magnetic perturbation,"Sensors, vol. 11, no. 12, pp. 11390-11414, Nov. 20.

[17] B. Shin et al., "Implementation and performance analysis of smartphonebased 3D PDR system with hybrid motion and heading classifier," 2014 IEEE/ION Position, Location and Navigation Symposium - PLANS 2014, Monterey, CA, 2014, pp. 201-204.

[18] L. Shi, Y. Zhao, G. Liu, S. Chen, Y. Wang and Y. Shi, "A Robust Pedestrian Dead Reckoning System Using Low-Cost Magnetic and Inertial Sensors," in IEEE Transactions on Instrumentation and Measurement, vol. 68, no. 8, pp. 2996-3003, Aug. 2019.

[19] Z. Li, C. Song, J. Cai, R. Hua and P. Yu, "An Improved Pedestrian Navigation System Using IMU and Magnetometer," 2017 International Conference on Computer Systems, Electronics and Control (ICCSEC), Dalian, 2017, pp. 1639-1642.

[20] H. Guo, M. Uradzinski, H. Yin, and M. Yu, "Indoor positioning based on foot-mounted IMU," Bull. Polish Acad. Sci. Tech. Sci., vol. 63, no. 3, pp. 629-634, Sep. 2015.

[21] Jaehyun Park, Yunki Kim and Jangmyung Lee, "Waist mounted Pedestrian Dead-Reckoning system," 2012 9th International Conference on Ubiquitous Robots and Ambient Intelligence (URAI), Daejeon, 2012, pp. 335-336.

[22] Seon-Woo Lee and K. Mase, "Activity and location recognition using wearable sensors," in IEEE Pervasive Computing, vol. 1, no. 3, pp. 2432, July-Sept. 2002.

[23] M. Garcia Puyol, D. Bobkov, P. Robertson and T. Jost, "Pedestrian Simultaneous Localization and Mapping in Multistory Buildings Using Inertial Sensors," in IEEE Transactions on Intelligent Transportation Systems, vol. 15, no. 4, pp. 1714-1727, Aug. 2014.

[24] J. Perul and V. Renaudin, "Learning Individual Models to Estimate the Walking Direction of Mobile Phone Users," in IEEE Sensors Journal, vol. 19, no. 24, pp. 12306-12315, 15 Dec.15, 2019.

[25] Z. Yang, Y. Pan and L. Zhang, "Hybrid orientation filter aided indoor tracking for pedestrians using a smartphone," 2017 13th IEEE International Conference on Control \& Automation (ICCA), Ohrid, 2017, pp. 107-112.

[26] D. Chen et al., "Smart Insole-Based Indoor Localization System for Internet of Things Applications," in IEEE Internet of Things Journal, vol. 6, no. 4, pp. 7253-7265, Aug. 2019.

[27] W. Chen et al., "Comparison of EMG-based and accelerometer-based speed estimation methods in pedestrian dead reckoning," J. Navigat., vol. 64, no. 2, pp. 265-280, 2011.

[28] W. Zhang, X. Li, D. Wei, X. Ji and H. Yuan, "A foot-mounted PDR system based on IMU/EKF+HMM+ZUPT+ZARU+HDR+compass algorithm," 2017 International Conference on Indoor Positioning and Indoor Navigation (IPIN), Sapporo, 2017, pp. 1-5.

[29] S. Asano, Y. Wakuda, N. Koshizuka and K. Sakamura, ”A robust pedestrian dead-reckoning positioning based on pedestrian behavior and sensor validity," Proceedings of the 2012 IEEE/ION Position, Location and Navigation Symposium, Myrtle Beach, SC, 2012, pp. 328-333.

[30] G. Trehard, S. Lamy-Perbal and M. Boukallel, "Indoor infrastructureless solution based on sensor augmented smartphone for pedestrian localisation," 2012 Ubiquitous Positioning, Indoor Navigation, and Location Based Service (UPINLBS), Helsinki, 2012, pp. 1-7.

[31]. Zizzo and L. Ren, "Position tracking during human walking using an integrated wearable sensing system," Sensors, vol. 17, no. 12, p. $2866,2017$.

[32] Y. Hsu, S. Huang, J. Perng, "Application of multisensor fusion to develop a personal location and 3D mapping system," Optik, vol. 172, pp. 328-339, 2018.

[33] Y. Wu, H. Zhu, Q. Du and S. Tang, "A Pedestrian Dead-Reckoning System for Walking and Marking Time Mixed Movement Using an SHSs Scheme and a Foot-Mounted IMU," in IEEE Sensors Journal, vol. 19, no. 5, pp. 1661-1671, 1 March1, 2019
[34] F. Inderst, F. Pascucci and M. Santoni, "3D pedestrian dead reckoning and activity classification using waist-mounted inertial measurement unit," 2015 International Conference on Indoor Positioning and Indoor Navigation (IPIN), Banff, AB, 2015, pp. 1-9.

[35] M. S. Lee, H. Ju, J. W. Song, and C. G. Park, "Kinematic model-based pedestrian dead reckoning for heading correction and lower body motion tracking," Sensors, vol. 15, no. 11, pp. 28129-28153, 2015.

[36] N. Choe, H. Zhao, S. Qiu, and Y. So, "A sensor-to-segment calibration method for motion capture system based on low cost MIMU," Measurement, vol. 131, pp. 490-500, Jan. 2019.

[37] A. Mikov, A. Moschevikin, A. Fedorov and A. Sikora, "A localization system using inertial measurement units from wireless commercial hand-held devices," International Conference on Indoor Positioning and Indoor Navigation, Montbeliard-Belfort, 2013, pp. 1-7.

[38] B. Shin et al., "Motion Recognition-Based 3D Pedestrian Navigation System Using Smartphone," in IEEE Sensors Journal, vol. 16, no. 18, pp. 6977-6989, Sept.15, 2016.

[39] L. Ruotsalainen, M. Kirkko-Jaakkola, J. Rantanen, and M. Mäkelä, "Error modelling for multi-sensor measurements in infrastructurefree indoor navigation," Sensors, vol. 18, no. 2, p. 590, 2018, doi: $10.3390 / \mathrm{s} 18020590$

[40] M. Edel and E. Köppe, "An advanced method for pedestrian dead reckoning using BLSTM-RNNs," 2015 International Conference on Indoor Positioning and Indoor Navigation (IPIN), Banff, AB, 2015, pp. $1-6$.

[41] M. A. Chattha and I. H. Naqvi, "PiLoT: A Precise IMU Based Localization Technique for Smart Phone Users," 2016 IEEE 84th Vehicular Technology Conference (VTC-Fall), Montreal, QC, 2016, pp. 1-5.

[42] M. Garcia Puyol, D. Bobkov, P. Robertson and T. Jost, "Pedestrian Simultaneous Localization and Mapping in Multistory Buildings Using Inertial Sensors," in IEEE Transactions on Intelligent Transportation Systems, vol. 15, no. 4, pp. 1714-1727, Aug. 2014.

[43] Xiaoli Meng, Shuyan Sun, Lianying Ji, Jiankang Wu and Wai-Choong Wong, "Displacement estimation in micro-sensor motion capture," 2010 IEEE International Conference on Systems, Man and Cybernetics, Istanbul, 2010, pp. 2611-2618.

[44] Y. Hsu, J. Wang and C. Chang, "A Wearable Inertial Pedestrian Navigation System With Quaternion-Based Extended Kalman Filter for Pedestrian Localization," in IEEE Sensors Journal, vol. 17, no. 10, pp. 3193-3206, 15 May15, 2017.

[45] M. N. Ibarra-Bonilla, P. Jorge Escamilla-Ambrosio, J. Manuel RamirezCortes and C. Vianchada, "Pedestrian dead reckoning with attitude estimation using a fuzzy logic tuned adaptive kalman filter," 2013 IEEE 4th Latin American Symposium on Circuits and Systems (LASCAS), Cusco, 2013, pp. 1-4.

[46] D. Wu, L. Xia, and J. Geng, "Heading estimation for pedestrian dead reckoning based on robust adaptive Kalman filtering,"Sensors, vol. 18,no. 6, p. 1970, Jun. 2018

[47] Q. Fan, H. Zhang, P. Pan, X. Zhuang, J. Jia, P. Zhang, Z. Zhao, G. Zhu, andY. Tang, "Improved pedestrian dead reckoning based on a robust adaptiveKalman filter for indoor inertial location system," Sensors, vol. 19, no. 2,p. 294, Jan. 2019

[48] J. Hightower, G. Borriello. "Particle filters for location es- timation in ubiquitous computing: A case study." Interna- tional Conference on Ubiquitous Computing, N. Davies, E. D. Mynatt, I. Siio, Eds., Berlin Heidelberg, Germany: Springer, pp. 88-106, 2004.

[49] M. Hardegger, D. Roggen, and G. Tröster, "3D ActionSLAM: Wearable person tracking in multi-floor environments," Pers. Ubiquitous Comput., vol. 19, no. 1, pp. 123-141, 2015.

[50] J. Borenstein, L. Ojeda, and S. Kwanmuang, "Heuristic reduction of gyro drift in IMU-based personnel tracking systems," Proc. SPIE, vol 7306, May 2009, Art. no. 73061H.

[51] H. Weinberg, "Using the ADXL202 in pedometer and personal navigation applications," Analog Devices, Norwood, MA, USA, Appl. Note AN-602, 2002, pp. 1-6, vol. 2, no. 2.

[52] J. W. Kim, H. J. Jang, D.-H. Hwang, and C. Park, "A step, stride and heading determination for the pedestrian navigation system," Positioning, vol. 3, nos. 1-2, pp. 273-279, 2004.

[53] J. Scarlet, "Enhancing the Performance of Pedometers Using a Single Accelerometer," Analog Devices AN-900 Application Note, 2005.

[54] V. Renaudin, M. Susi, and G. Lachapelle, "Step length estimation using handheld inertial sensors," Sensors, vol. 12, no. 7, pp. 8507-8525, 2012.

[55] H. Wang, S. Sen, A. Elgohary, M. Farid, M. Youssef, and R. R. Choudhury, "No need to war-drive: Unsupervised indoor localization," in Proc. 10th Int. Conf. Mobile Syst., Appl., Services (MobiSys), Jun. 2012, pp. 197-210. 
[56] articlebergmann2011bodyBergmann, JHM and McGregor, AH., "Bodyworn sensor design: what do patients and clinicians want?"' in Annals of biomedical engineering,vol. 39, no. 9, pp. 2299-2312, 2011.

[57] Hussain, G.; Jabbar, M.; Cho, J.D.; Bae, S. "Indoor Positioning System: A New Approach Based on LSTM and Two Stage Activity Classification," Electronics2019 ,8, 375.

[58] H. Zhang, W. Yuan, Q. Shen, T. Li and H. Chang, "A Handheld Inertial Pedestrian Navigation System With Accurate Step Modes and Device Poses Recognition," in IEEE Sensors Journal, vol. 15, no. 3, pp. 14211429, March 2015.

[59] Y. Wu, H. Zhu, Q. Du and S. Tang, "A Pedestrian Dead-Reckoning System for Walking and Marking Time Mixed Movement Using an SHSs Scheme and a Foot-Mounted IMU," in IEEE Sensors Journal, vol. 19, no. 5, pp. 1661-1671, 1 March1, 2019.

[60] Z. Sun, X. Mao, W. Tian, and X. Zhang, "Activity classification and dead reckoning for pedestrian navigation with wearable sensors," Meas. Sci. Technol., vol. 20, no. 1, p. 015203, Nov. 2008.

[61] Y. Guo, Q. LIU, X. Ji, S. Wang, M. Feng, Y. Sun, "Multimode Pedestrian Dead Reckoning Gait Detection Algorithm Based on Identification of Pedestrian Phone Carrying Position," Mobile Information Systems, 2019.

[62] M. Angermann and P. Robertson, "FootSLAM: Pedestrian Simultaneous Localization and Mapping Without Exteroceptive Sensors - Hitchhiking on Human Perception and Cognition," Proceedings of the IEEE, vol. 100, pp. 1840-1848, May 2012.

[63] S. Kaiser and E. M. Diaz, "PocketSLAM based on the principle of the FootSLAM algorithm," 2015 International Conference on Localization and GNSS (ICL-GNSS), Gothenburg, 2015, pp. 1-5.

[64] M. Hardegger, D. Roggen, S. Mazilu and G. Tröster, "ActionSLAM: Using location-related actions as landmarks in pedestrian SLAM," 2012 International Conference on Indoor Positioning and Indoor Navigation (IPIN), Sydney, NSW, 2012, pp. 1-10.

[65] J. Prieto, S. Mazuelas and M. Z. Win, "Context-Aided Inertial Navigation via Belief Condensation," in IEEE Transactions on Signal Processing, vol. 64, no. 12, pp. 3250-3261, June15, 2016.

[66] T. Do, R. Liu, C. Yuen, M. Zhang and U. Tan, "Personal Dead Reckoning Using IMU Mounted on Upper Torso and Inverted Pendulum Model," in IEEE Sensors Journal, vol. 16, no. 21, pp. 7600-7608, Nov.1, 2016.

[67] W. Elloumi, A. Latoui, R. Canals, A. Chetouani and S. Treuillet, "Indoor Pedestrian Localization With a Smartphone: A Comparison of Inertial and Vision-Based Methods," in IEEE Sensors Journal, vol. 16, no. 13, pp. 5376-5388, July1, 2016.

[68] L. Van Nguyen and H. M. La, "Real-Time Human Foot Motion Localization Algorithm With Dynamic Speed," in IEEE Transactions on Human-Machine Systems, vol. 46, no. 6, pp. 822-833, Dec. 2016.

[69] C. Randell, C. Djiallis and H. Muller, "Personal position measurement using dead reckoning," Seventh IEEE International Symposium on Wearable Computers, 2003. Proceedings., White Plains, NY, USA, 2003, pp. 166-173.

[70] S. Cardarelli, P. di Florio, A. Mengarelli, A. Tigrini, S. Fioretti and F. Verdini, "Magnetometer-Free Sensor Fusion Applied to Pedestrian Tracking: A Feasibility Study," 2019 IEEE 23rd International Symposium on Consumer Technologies (ISCT), Ancona, Italy, 2019, pp. 238242.

[71] N. Yu, Y. Li, X. Ma, Y. Wu and R. Feng, "Comparison of Pedestrian Tracking Methods Based on Foot- and Waist-Mounted Inertial Sensors and Handheld Smartphones," in IEEE Sensors Journal, vol. 19, no. 18, pp. 8160-8173, 15 Sept.15, 2019.

[72] J. Kuan, X. Chen, X. Niu, "Research on Robust PDR Algorithm Based on Smart Phone," China Satellite Navigation Conference (CSNC) 2018 Proceedings vol. 499, pp. 673-684, 2018.

[73] C. Randell, C. Djiallis and H. Muller, "Personal position measurement using dead reckoning," Seventh IEEE International Symposium on Wearable Computers, 2003. Proceedings., White Plains, NY, USA, 2003, pp. 166-173.

[74] Young Soo Suh and S. Park, "Pedestrian inertial navigation with gait phase detection assisted zero velocity updating," 2009 4th International Conference on Autonomous Robots and Agents, Wellington, 2009, pp. 336-341.

[75] H. Zhao et al., "Heading Drift Reduction for Foot-Mounted Inertial Navigation System via Multi-Sensor Fusion and Dual-Gait Analysis," in IEEE Sensors Journal, vol. 19, no. 19, pp. 8514-8521, 1 Oct.1, 2019, doi: 10.1109/JSEN.2018.2866802.

[76] A. Poulose, O. S. Eyobu and D. S. Han, "An Indoor Position-Estimation Algorithm Using Smartphone IMU Sensor Data," in IEEE Access, vol. 7, pp. 11165-11177, 2019, doi: 10.1109/ACCESS.2019.2891942.
[77] S. Qiu et al., "Inertial/magnetic sensors based pedestrian dead reckoning by means of multi-sensor fusion," in Information Fusion, vol. 39, pp. 108-119, 2018. 